\title{
Identification of Detailed Time-Frequency Components in Somatosensory Evoked Potentials
}

\author{
Zhiguo Zhang, Member, IEEE, Keith D. K. Luk, and Yong Hu, Member, IEEE
}

\begin{abstract}
Somatosensory evoked potential (SEP) usually contains a set of detailed temporal components measured and identified in time domain, providing meaningful information on physiological mechanisms of the nervous system. The purpose of this study is to reveal complex and fine time-frequency features of SEP in time-frequency domain using advanced time-frequency analysis (TFA) and pattern classification methods. A high-resolution TFA algorithm, matching pursuit (MP), was proposed to decompose a SEP signal into a string of elementary waves and to provide a timefrequency feature description of the waves. After a dimension reduction by principle component analysis (PCA), a density-guided K-means clustering was followed to identify typical waves existed in SEP. Experimental results on posterior tibial nerve SEP signals of 50 normal adults showed that a series of typical waves were discovered in SEP using the proposed MP decomposition and clustering methods. The statistical properties of these SEP waves were examined and their representative waveforms were synthesized. The identified SEP waves provided a comprehensive and detailed description of time-frequency features of SEP.
\end{abstract}

Index Terms-Density estimation, K-means clustering, matching pursuit, somatosensory evoked potentials, time-frequency analysis.

\section{INTRODUCTION}

$\mathbf{S}$ OMATOSENSORY evoked potential (SEP) is the electrical response of the central nervous system to an electrical stimulation of a peripheral nerve. SEP has been widely used in electrophysiological diagnosis and intraoperative monitoring because it is capable of providing meaningful information on physiological mechanisms of the nervous system [1]-[4]. In previous studies, a number of stable temporal components were validated to exist in SEP and they reflect sequential activation of neural structures along the somatosensory pathways [1]-[7]. These detailed temporal components were generally identified by measuring latencies and amplitudes of a set of small onsets, peaks, and notches in time domain.

Manuscript received July 22, 2009; revised December 11, 2009; accepted February 02, 2010. First published March 08, 2010; current version published June 09, 2010. This work was supported in part by a grant from the Research Grants Council of the Hong Kong SAR, China (GRF HKU 7130/06E), in part by the Biomedical Engineering Centre (BMEC) of the University of Hong Kong, and in part by The University of Hong Kong CRCG Seed Fund.

Z. G. Zhang was with the Department of Orthopaedic and Traumatology, the University of Hong Kong, Duchess of Kent Children's Hospital, Hong Kong, China. He is now with the Department of Electrical and Electronic Engineering, the University of Hong Kong, Pokfulam, Hong Kong (e-mail: zgzhang@eee. hku.hk)

K. D. K. Luk and Y. Hu are with the Department of Orthopaedic and Traumatology, the University of Hong Kong, Duchess of Kent Children's Hospital, Hong Kong (e-mail: hrmoldk@hku.hk; yhud@hkusua.hku.hk).

Digital Object Identifier 10.1109/TNSRE.2010.2043856
The identification and measurement of SEP components can also be performed in time-frequency domain by time-frequency analysis (TFA) methods. Recently, it was found that TFA of SEP provided an earlier and more sensitive indication of neural injury than time domain measurements, and thus it is suggested that TFA of SEP should be useful in preventing spinal cord injury during surgery [8]-[12]. TFA of a SEP signal leads to a time-frequency distribution (TFD), describing the energy or power of the signal as a 2-D function of time and frequency. Generally, the TFD of a normal SEP signal exhibits a distinct peak in the time-frequency domain, and the time-frequency features of the SEP signal are extracted as the measurements of the main peak in the TFD, including the peak power, peak time, and peak frequency [9]-[12]. Compared with the temporal components of SEP, the time-frequency features are more stable and easily-identifiable, and they present rapid changes in time-frequency domain when deficits happen in spinal cord function [8]-[12].

Currently, only the time-frequency features associated with the main peak in the TFD of SEP were examined in detail. However, there are many other minute peaks and features existed in the TFD and their features have not been studied yet. Although the short-time Fourier transform (STFT) was recommended in [9]-[12] as an appropriate method for SEP analysis among various TFA methods, the minute peaks revealed by STFT heavily depend on the selection of analysis window, making it difficult to obtain reliable and stable time-frequency features as references. In [13]-[15], multiresolution wavelet analysis was proposed to expand an evoked potential into a set of coarse and detailed components. The wavelet analysis provided an alternative way, i.e., time-frequency decomposition, to analyze SEP signals, but it could not offer a time-frequency parameter description of the decomposed components and thus it was difficult to establish reference values to assess the SEP.

This study aims to reveal complex and fine time-frequency features of SEP using advanced TFA and pattern classification methods. Firstly, to overcome the limitations of STFT and wavelet analysis, a high-resolution TFA method, matching pursuit (MP), was adopted for SEP analysis. The MP method decomposes a signal into a series of time-frequency components (TFCs) from a large and redundant dictionary in an iterative fashion [16]. More importantly, the MP algorithm is able to provide a straightforward parameter description of the decomposed TFCs. In addition, the MP method is very robust in the presence of noise, so it is suitable for dealing with SEP accompanied with heavy background noise [17]. All these merits make the MP algorithm a powerful tool in processing of various biomedical signals, such as electroencephalography 
(EEG) [17], otoacoustic emission (OAE) [18], and visual evoked potential (VEP) [19]. In this paper, the Gabor dictionary was used in the MP decomposition and each Gabor-type TFC was described by five parameters, including the latency, frequency, time span, amplitude, and phase. Secondly, we used pattern recognition methods to identify stable and representative time-frequency features from a large number of TFCs decomposed from SEP signals under study. To fulfill this purpose, clustering analysis was applied to group the TFCs into a finite number of clusters, and each cluster of TFCs had similar time-frequency parameters (features). The K-means clustering algorithm was adopted, and the probability density function (PDF) of TFCs was estimated to provide initial centroids for $\mathrm{K}$-means. Because it was difficult to perform a clustering in a 5-D space (a Gabor-type TFC was described by five features), principle component analysis (PCA) was applied to reduce the dimension of the feature space prior to the clustering [20].

SEP signals recorded from fifty patients undergoing scoliosis surgery were studied in the paper. Five clusters of TFCs were identified, and each cluster represented a typical SEP wave embodying specific and stable time-frequency features of normal SEPs. The statistical properties of the SEP waves were calculated to give reference values for normal SEP signals. Representative waveforms of these SEP waves were synthesized accordingly, and their sum yielded a synthetic normal SEP signal with typical time-frequency features. Moreover, the correlations between different SEP waves were calculated to investigate the dependencies between them. We also showed that the time-frequency features identified in our study were in good accordance with SEP's temporal components revealed in previous studies. All these results together presented an integrated and clear picture on the time-frequency nature of normal SEPs, and they provided more detailed time-frequency features than conventional TFA methods.

\section{MATERIALS AND MethodS}

\section{A. Experimental Procedure and Data Collection}

Fifty patients undergoing scoliosis surgery were included in the study ( 38 female and 12 male). Their age ranged from 11 to 18 years (mean 15 years). All patients received general anaesthesia, induced by thiopentone (thiopental) $(0.4 \mathrm{mg} / \mathrm{kg})$ or fentanyl $(1-2 \mathrm{mg} / \mathrm{kg})$. Isoflurane $(0.5 \%-0.8 \%)$ and nitrous oxide/ oxygen (typically 60:40\%) were used to maintain the anaesthesia. None of the patients had any neurological deficit before, immediately after, or at the two months assessment following the surgery.

A pair of stimulating electrodes was applied over the posterior tibial nerve (PTN) behind the medial malleoli to elicit SEP, and the constant current stimulation was in the range of 10-30 mA. Single pulse stimulation with a frequency between 5.1 and $5.7 \mathrm{~Hz}$ and duration of $0.3 \mathrm{~ms}$ was used [21]. The SEP signals were collected over $\mathrm{Cz}^{\prime}(2 \mathrm{~cm}$ posterior to $\mathrm{Cz}, 10-20$ international system of EEG electrode placement) versus the Fz of the 10-20 system using subcutaneous needle electrodes. An intraoperative spinal cord monitoring system (Nicolet Viking IV, Nicolet Biomedical, Madison, WI) was employed to record the responses. A $20-3000 \mathrm{~Hz}$ bandpass filter and automatic arte- fact rejection was applied to reduce noise. The sweep time of SEP recording was $100 \mathrm{~ms}$. To eliminate possible stimulation artefacts, the first $10 \mathrm{~ms}$ of each $100 \mathrm{~ms}$ trial were discarded and the remaining $90 \mathrm{~ms}$ of data were kept for further analysis. Each SEP signal was obtained by ensemble average of 100 trials to improve the SNR. The averaged SEP were measured alternately during left or right sided stimulation every 15-20 min during noncritical procedures such as incision, and continuously during critical surgical procedures or if abnormal SEP occurred. The averaged SEP signals were then analysed on the Pentium 4 PC platform (3.2 GHz, 1 GB RAM) using MATLAB software (version 7.0, Mathworks, Natick, MA).

\section{B. Matching Pursuit}

Given a discrete-time SEP signal $x(n)$, the MP method expands $x(n)$ into a sum of TFCs

$$
x(n)=\sum_{m=1}^{M} g_{m}(n)+e(n)
$$

where $g_{m}(n)$ is the $m$ th TFC, $M$ is the number of decomposed TFCs, and $e(n)$ is the decomposition residue. The MP decomposition is generally achieved in an iterative manner, and interested readers are referred to [16]-[19] for more details.

In the MP method, the TFCs are chosen from a redundant dictionary, and different dictionaries will result in different decomposition results. The Gabor dictionary was used in our study due to its simplicity, completeness, and good time-frequency localizations [16], [17]. A more complicated dictionary, such as the chirplet dictionary [19], describes a TFC with more parameters, and it may give a better representation if SEP's underlying time-frequency components match the dictionary well. However, the true shapes of SEP components are unknown, and a more complicated dictionary leads to a heavier computational complexity. In the MP algorithm, the interpretation on decomposition results is more important than selection of dictionary [16], [17]. Thus, the Gabor dictionary is enough to describe most signals, including SEP.

A Gabor-type TFC has the form as

$$
g(n)=a e^{-\pi[(n-t) / s]^{2}} \cos [2 \pi f(n-t)+\phi]
$$

where $t, f, s, a$, and $\phi$ are, respectively, the latency, frequency, span, amplitude, and phase. In the Gabor function, $e^{-\pi[(n-t) / s]^{2}}$ is the waveform envelope with center at time $t$ and span described by $s$. The parameter $t$ is the waveform's latency, which is defined as the time duration from the stimulus onset to the maximum of the waveform envelope. It can be seen that a Gabortype TFC is generated by translating (with $t$ ), modulating (with $f$ ), dilating (with $s$ ), scaling (with $a$ ), and phase-shifting (with $\phi$ ) an envelope function. Therefore, the latency $t$, the frequency $f$, the span $s$, the amplitude $a$, and the phase $\phi$ constitute a 5-D parameter vector $\boldsymbol{u}=[t, f, s, a, \phi]^{T}$ to characterize a Gabortype TFC.

\section{Feature Selection and Dimension Reduction}

We next grouped all the TFCs decomposed from 50 SEP signals into several clusters and extracted representative time-frequency features of each cluster. Due to the curse of dimension- 
ality [20], it was difficult to cluster these TFCs in a 5-D feature space and thus a dimension reduction was a crucial procedure prior to the clustering. On the other hand, if the dimensionality was too low, say, one or two, the number of clusters might be underestimated. By comparing the complexity and accuracy, we chose to use three features in the clustering.

In our study, the dimensionality was reduced by principal component analysis (PCA). Compared with other dimension reduction techniques like projection pursuit and factor analysis, PCA has a simple and efficient implementation and its effectiveness has been validated in numerous applications [22]. PCA aims to reduce the dimensionality of the data while retaining as much as possible of the information (variance) of the original data. Consider an original $d \times N$ data set $\boldsymbol{X}$, where $N$ is the number of sample vectors and $d$ is the dimension of sample vectors (or number of variables), with $d \times 1$ mean vector $\boldsymbol{\mu}_{X}$ and $d \times d$ covariance matrix $C_{\boldsymbol{X} \boldsymbol{X}}$. The eigenvalue decomposition of $\boldsymbol{C}_{\boldsymbol{X} \boldsymbol{X}}, \boldsymbol{C}_{\boldsymbol{X} \boldsymbol{X}}=\boldsymbol{U} \Lambda \boldsymbol{U}^{T}$, yields a diagonal matrix $\Lambda$ with d eigenvalues (sorted in descending order) of $\boldsymbol{C}_{\boldsymbol{X} \boldsymbol{X}}$ and an orthogonal matrix $\boldsymbol{U}$ whose columns consist of d eigenvectors. By a linear transformation $\boldsymbol{Y}=\boldsymbol{U}^{T}\left(\boldsymbol{X}-\boldsymbol{\mu}_{\boldsymbol{X}}\right)$, PCA forms a new set of data $\boldsymbol{Y}$, whose rows are principal components orthogonal to each other. The resultant first principal component has the largest variance and so on. We can only preserve $l(l<d)$ principal components of $\boldsymbol{Y}$ with large variances and discarded others, which was shown to be the best approximation (in a sense of minimum mean squared error) to the original data in a reduced dimension [22].

Among the five parameters of a TFC, latency $t$ and frequency $f$ were more important than others because they determined the position of TFCs in the time-frequency domain. Thus, PCA was only used for other three parameters: $s, a$, and $\phi$. To eliminate the influence of different scales, parameters $s, a$, and $\phi$ were first standardized to have mean zero and standard deviation (SD) one. After the PCA, the first principal component (PC1, with symbol $p$ ) was used for the following clustering, and the feature vector to describe a TFC was reduced to $\boldsymbol{v}=[t, f, p]^{T}$. That is, the TFCs were clustered in a 3-D space according to their latencies, frequencies, and $\mathrm{PC} 1$ values.

\section{Density-Guided K-Means Clustering}

To simplify the clustering, the main TFCs, which accounted for the largest energy in each SEP signal, were first categorized as an individual cluster. We later showed that these main TFCs shared similar time-frequency features and were associated with the main peaks of TFDs obtained by other TFA methods. In the following study, we mainly focused on the clustering of $N$ sub-TFCs, i.e., all the TFCs decomposed from 50 subjects except 50 main TFCs. The classical K-means clustering method was adopted here mainly due to its simplicity and effectiveness. Also, compared with other popular clustering methods, $\mathrm{K}$-means doesn't need to know the data distribution (as compared with Gaussian mixture method) and its results are more robust and easily-interpretable (as compared with hierarchical clustering) [23]. Given the number of clusters $(K)$ and initial centroids of clusters, the K-means algorithm assigned each TFC to the nearest cluster centroid. However, the K-means algorithm is very sensitive to the selection of the number of clusters and the initial cluster centroids, whose different values may lead to totally different clustering results. A commonly-used initial guess for $\mathrm{K}$-means is obtained by placing cluster centroids at the peaks (local maxima with higher values than neighbouring areas) in the joint probability density function (PDF) of the samples [24]-[26]. Therefore, we first estimated the joint PDF of TFCs in the 3-D feature space in order to provide necessary guide for the K-means algorithm. A multidimensional joint PDF was estimated by the kernel density estimation algorithm using Gaussian kernels as

$$
P(\boldsymbol{v})=\sum_{i=1}^{N} \frac{1}{(2 \pi)^{N / 2} \operatorname{det}(\boldsymbol{H})} e^{-\left[\left(\boldsymbol{v}-\boldsymbol{v}_{i}\right)^{T} \boldsymbol{H}^{-1}\left(\boldsymbol{v}-\boldsymbol{v}_{i}\right)\right] / 2}
$$

where $N$ was the number of sub-TFCs, $\boldsymbol{H}$ was the bandwidth matrix to adjust the smoothness of the PDF and $\operatorname{det}(\boldsymbol{H})$ was the determinant of $\boldsymbol{H}$. The bandwidth matrix $\boldsymbol{H}$ should be carefully selected to avoid any under-smoothed or over-smoothed PDF. As suggested in [27], the bandwidth matrix $\boldsymbol{H}$ was chosen as a diagonal matrix $\boldsymbol{H}=\operatorname{diag}\left(\left[h^{(t)}, h^{(f)}, h^{(p)}\right]\right)$, where $h^{(\gamma)}(\gamma$ indicated $t, f$, or $p$ ) was optimally calculated to minimize the asymptotic mean integrated squared error (AMISE) in the respective dimension. More precisely, the bandwidth parameter $h^{(\gamma)}$ was obtained as

$$
h^{(\gamma)}=\left[\frac{4}{(d+2) N}\right]^{1 /(d+4)} \sigma^{(\gamma)}
$$

where $d$ was the dimension of the feature vector $(d=3$ in this study) and $\sigma^{(\gamma)}$ was the SD in dimension $\gamma$. A simple estimate of $\sigma^{(\gamma)}$ was given as

$$
\hat{\sigma}^{(\gamma)}=\operatorname{median}\left(\left|\gamma_{i}-\bar{\gamma}\right|\right) / 0.6745
$$

where $\bar{\gamma}$ was the median of $\gamma_{i}, i=1,2, \cdots, N$.

In the feature space, the joint PDF showed some peak areas, where the TFCs having similar features were concentrated. After detecting these PDF peaks, the number of peaks was adopted as the number of clusters and the locations of peaks were used as the initial centroids for the K-means clustering.

\section{RESULTS}

\section{A. Time-Frequency Analysis of SEP}

An example of PTN-SEP signal and its TFD are illustrated in Fig. 1. The TFD was obtained by a short-time Fourier transform (STFT) with a 40 ms Hanning window. It was validated in [12] that, the main peak in the TFD can provide more reliable monitoring parameters than conventional temporal components, such as P37 and N45.

Unlike the STFT method, the MP algorithm does not directly present an energy distribution in time-frequency domain but decomposes the signal into a series of TFCs described by a set of time and frequency parameters. In our study, MP decomposition was performed until decomposed TFCs explained $99 \%$ of the energy of each signal. Five highest energy TFCs decomposed from the SEP signal in Fig. 1 are illustrated in Fig. 2(a). The Wigner-Ville distribution (WVD) of each decomposed TFC was 
(a)

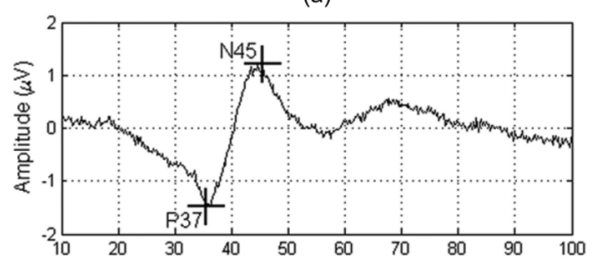

(b)

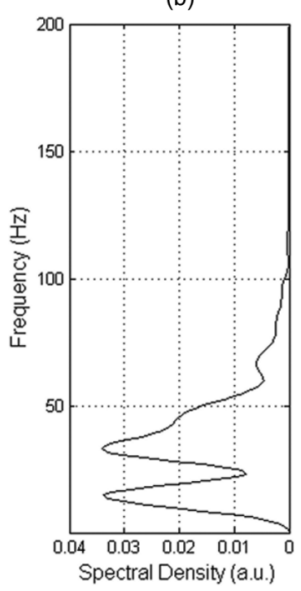

(c)

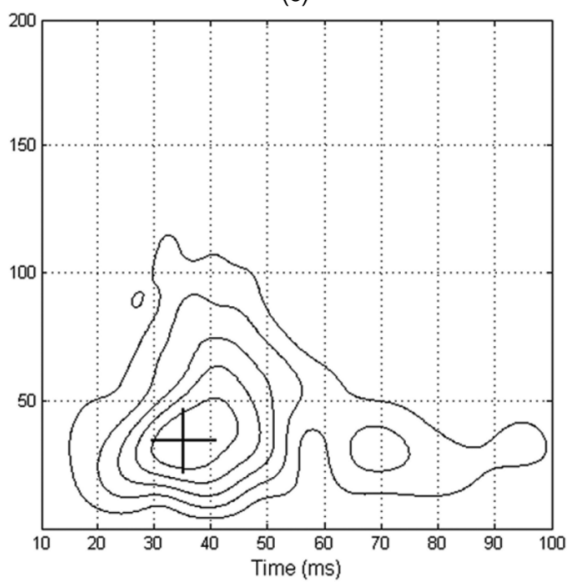

Fig. 1. The waveform, periodogram, and STFT-based TFD of an example of PTN-SEP signal. (a) A typical PTN-SEP waveform; (b) the periodogram calculated with fast Fourier transform; (c) STFT with a $40 \mathrm{~ms}$ Hanning window. The cross signs in (a) indicate the P37/N45 temporal components, and the cross in (c) denotes the main peak in the TFD.

(a)

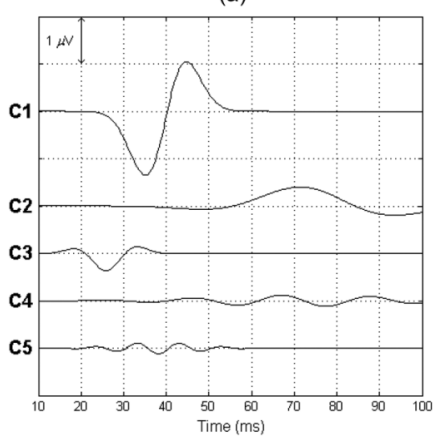

(b)

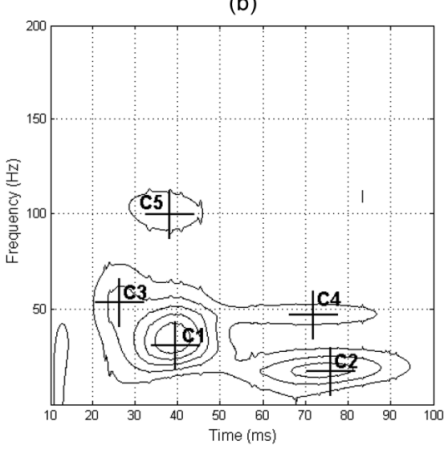

Fig. 2. TFCs from an example of PTN-SEP using MP decomposition and their TFD. (a) Five highest energy TFCs (C1-C5) are shown; (b) the TFD of the SEP signal was obtained by summing the WVDs of all decomposed TFCs. The cross signs in (b) denote the latencies and frequencies of five highest energy TFCs in the time-frequency domain.

also estimated, and the sum of all the WVDs gave a straightforward energy distribution of the SEP signal in the time-frequency domain [17], as shown in Fig. 2(b).

Apart from several high energy TFCs, such as those shown in Fig. 2, a majority of TFCs had very low energies and contributed little to the energy distribution of the whole signal. To facilitate the following clustering, we only considered the TFCs with relative energy, which was calculated as the ration between the energy of a TFC and the energy of the signal, greater than $1 \%$. In addition, those TFCs with too low frequency $(f<1 \mathrm{~Hz})$ or too short time $\operatorname{span}(s<1 \mathrm{~ms})$ were discarded as spurious components originated from baseline shift or impulsive noise.

After these filtering procedures, a total of 164 TFCs from 50 SEP signals were used for further clustering and statistical analysis. As mentioned in the previous section, 50 main TFCs of 50 subjects were first regarded as a cluster, and we only considered the clustering of 114 sub-TFCs.

Fig. 3 presents the histograms (normalized by the number of main or sub-TFCs) and 1-D PDFs of five MP parameters $(t, f, s, a, \phi)$ of the main TFCs and sub-TFCs. The 1-D PDFs were estimated using the kernel density estimation method in (3)-(5). The distinct peaks in PDFs of main TFCs implied that the main TFCs had clear and similar characteristics, especially their latencies (around $40 \mathrm{~ms}$ ) and frequencies (around $50 \mathrm{~Hz}$ ). On the other hand, the information provided by histograms and PDFs of sub-TFCs was relatively vague. No evident characteristic, or more than one evident characteristic were observed in the histograms and PDFs of sub-TFCs. For instance, two peaks around $40 \mathrm{~ms}$ and $80 \mathrm{~ms}$ existed in the PDF of latency of sub-TFCs. Therefore, these sub-TFCs should be further categorized into several individual clusters to present straightforward and useful features.

\section{B. Identification of SEP Waves}

Prior to the clustering, PCA was conducted to reduce the dimension and the joint PDF of the sub-TFCs was estimated to give initial conditions to the K-means clustering. Fig. 4(a) showed the 3-D joint PDF of the sub-TFCs in the latency-frequency-PC1 feature space. In the joint PDF, all the local peaks in the 3-D feature space were detected as candidates for initial centroids of clusters. A total of 25 peaks were discovered, but, of course, not all of them indicated a potential cluster. By checking the PDF values of these peaks, we found that four peaks (P1-P4, as indicated by cross signs in Fig. 4) had relatively large PDF values $(100 \%, 92 \%, 84 \%$, and $77 \%$ of the maximum PDF value, $\left.P_{\max }\right)$, while other peaks have values ranging from $18 \%$ to $49 \%$ of $P_{\max }$. By plotting these peaks values in descending order, as shown in Fig. 4(c), we can see that P1-P4 have distinctly larger values than others. So, the four PDF peaks, P1-P4, were used as initial centroids for K-means, and the number of clusters was determined as four.

The K-means clustering was performed for sub-TFCs in the 3-D latency-frequency-PC1 feature spaces and the results were shown in Fig. 5. Four clusters of sub-TFCs were identified and the numbers of sub-TFCs in each cluster were respectively 35 , 41, 19, and 19. These numbers were further adjusted because some SEP signals contained more than one TFC in a cluster. If a cluster had two or more TFCs from one signal, only the TFC with the highest energy was retained, and other TFCs with smaller energies were discarded. After the adjustment, the numbers of TFCs in the four clusters were 34, 32, 18, and 18, respectively. The 50 main TFCs were also shown in Fig. 5 and they were naturally categorized into one cluster, named as wave I. Four clusters of sub-TFCs were labeled as waves II-V. That is, five representative SEP waves were identified in PTN-SEP of normal adults. In summary, the clustering of Gabor-type TFCs include following steps: 1) selection of the main wave I with the maximal power; 2 ) dimension reduction (5-D to $3-\mathrm{D})$ by $\mathrm{PCA}$; 3) removal of TFCs with too low power; 4) density estimation 

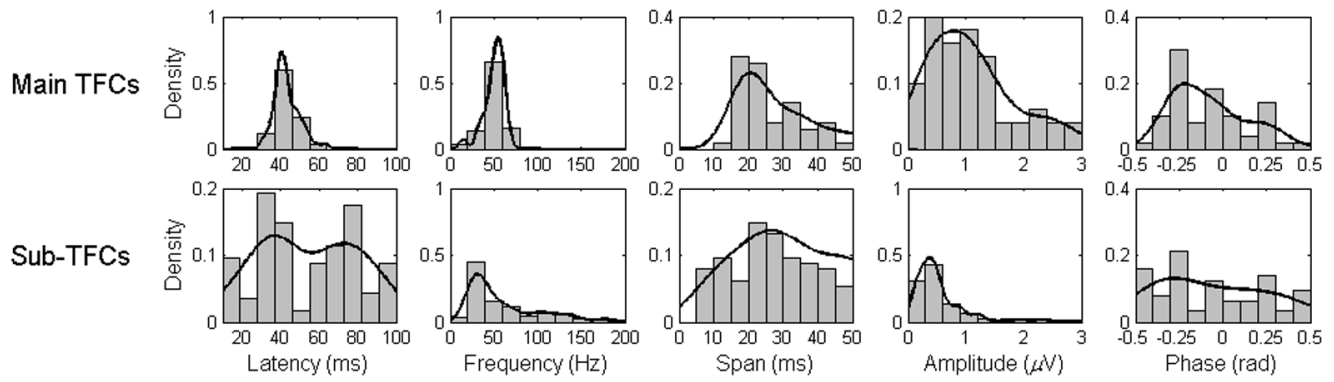

Fig. 3. Histograms and 1-D PDFs of MP parameters of main TFCs and sub-TFCs from 50 PTN-SEP signals. The histograms are normalized by the number of main or sub-TFCs. The total number of main TFCs is 50 and the total number of sub-TFCs is 114.

(a)
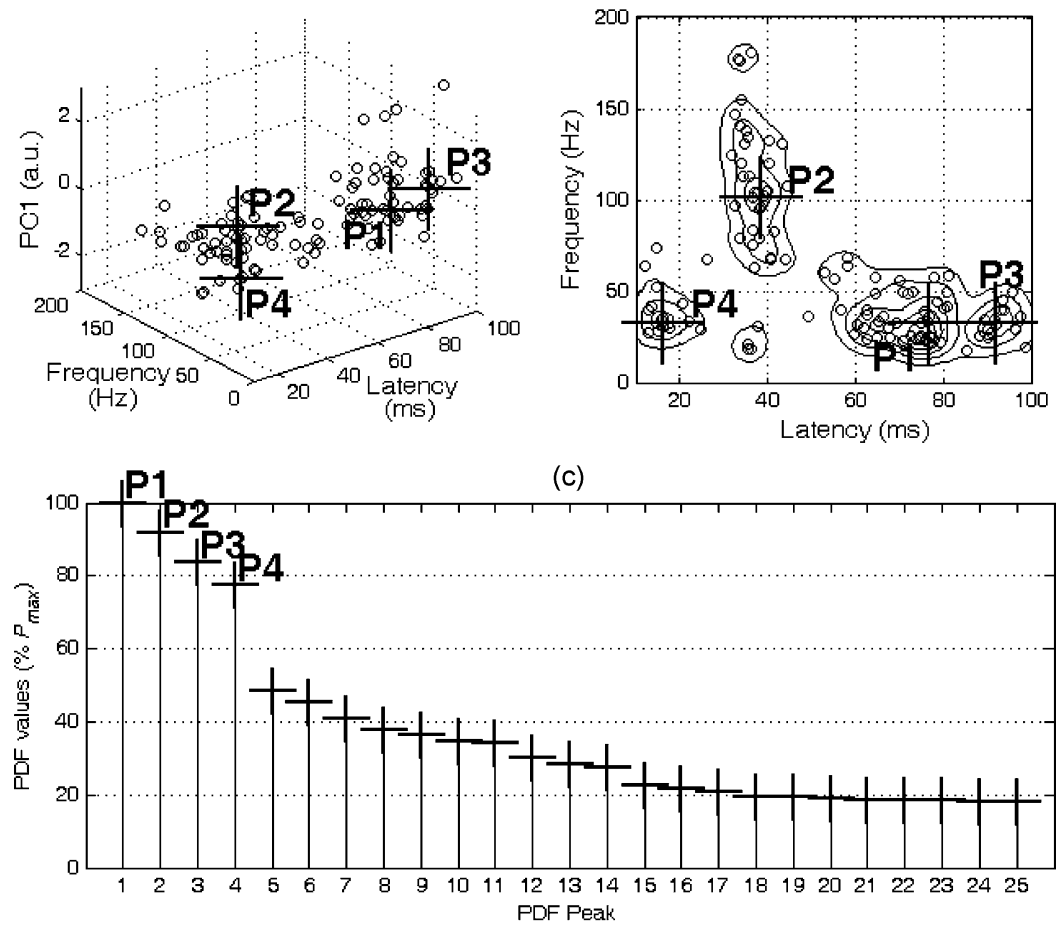

(b)

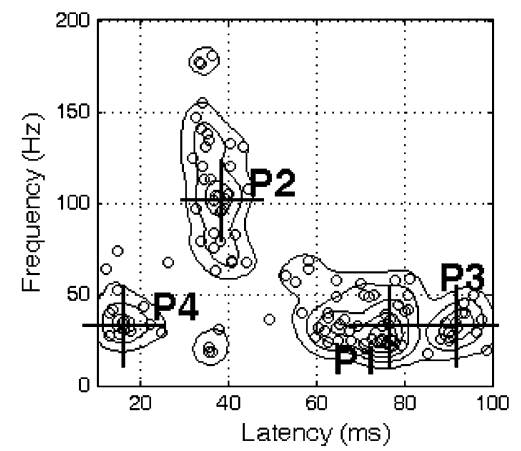

(c)

Fig. 4. Distribution and 3-D joint PDF of sub-TFCs. (a) Distributions of sub-TFCs in the latency-frequency-PC1 feature space; (b) the projections of the 3-D join PDF on 2-D latency-frequency feature spaces; (c) PDF values of peaks detected in the 3-D joint PDF. The circles denote the TFCs, and the cross signs indicate the PDF peaks used as initial centroids for clustering.

and K-means clustering; 5) removal of redundant TFCs from one subject in one cluster.

\section{Statistical Analysis of SEP Waves}

We further study the statistical characteristics of these identified SEP waves. The histograms and 1-D PDFs of wave's parameters are illustrated in Fig. 6. It can be seen that most parameters had prominent characteristics, especially the latency and frequency. The characteristics of span, amplitude, and phase, were not very significant, because the three parameters were not directly and individually used in clustering. The mean, SD, and coefficient of variation (CV) of the parameters of five waves are listed in Table I for quantitative assessment. The $\mathrm{CV}$ values were calculated as $\mathrm{CV}=100 * \mathrm{SD} /$ Mean and were used to measure and compare the variability between different sets of parameters with different scales. Since CV is not suitable for parameters having negative values or having mean values close to zero, the
$\mathrm{CV}$ values of phase were not included in Table I. We also calculated the average CVs of all parameters for each wave and average CVs of all waves for each parameter. The former average CVs showed that the latency had the smallest variability and the amplitude had the largest variability, while the latter showed that waves I, II, and III had relatively smaller parameter variability than waves IV and V. Interpretation of these observations will be discussed in the next section.

Next, for the purpose of identifying the dependency between different parameters, the correlation coefficients between parameters of each wave were calculated and listed in Table II. Similarly, the correlation coefficients between different waves for each parameter were calculated and listed in Table III to investigate the associations between different waves in one SEP signal. The latter correlation calculation was a little more complicated than the former, because not all SEP signals consisted of all the five waves. If we wanted to calculate the correlation 
(a)

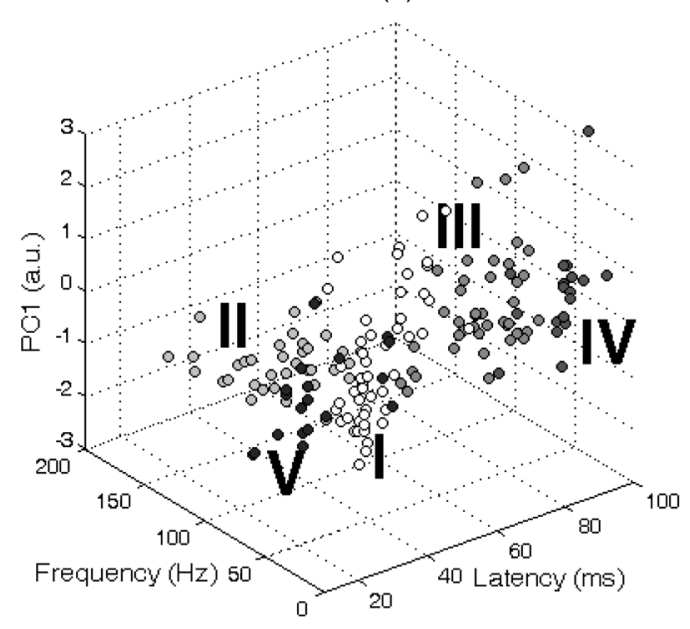

(b)

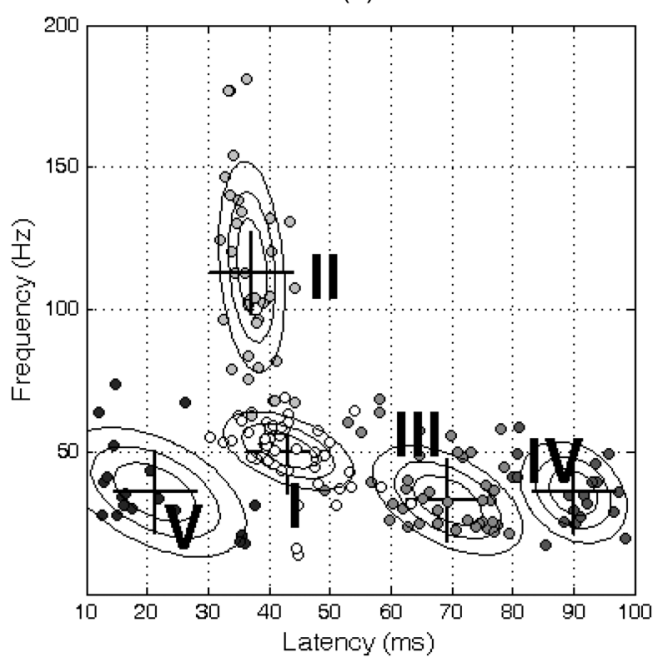

Fig. 5. Distribution and clustering results of TFCs. (a) Distribution and clustering results of TFCs in 3-D space, where different grey levels denote different clusters of TFCs; (b) distribution and clustering results of TFCs in time-frequency domain, where the contour lines denote the Gaussian probability densities calculated from the mean and covariance values in Tables I and II.

between some parameters of two waves, say, latencies of waves II and III, we first found the SEP signals containing both waves II and III from all the 50 signals. Then, the latencies of waves II and III in these signals formed two vectors for calculation of correlation coefficient.

We can read from Table II that: 1) the latency and frequency showed some considerable negative correlations in all the five waves; 2) the amplitudes had negative correlations with frequency and span in most waves to some extent. On the other hand, Table III told us that: 1) some large correlations were observed between waves III and IV for all parameters except phase; 2) the latencies of waves I and II had some large positive correlations; 3) wave $\mathrm{V}$ showed a number of strong correlations with other waves in many parameters.

The results in Table II implied that the latency and frequency of one SEP wave were not independently distributed. As a result, we calculated the covariance matrix of latency and frequency and showed their joint normal distribution in the time-frequency domain. It can be seen from Fig. 5(b) that all these waves had clear distribution patterns in the time-frequency domain. Generally, the frequencies decreased a little with the increase of latencies, representing the negative correlations between the two parameters. Other correlations observed in Tables II and III were discussed in the next section.

\section{Synthesized SEP Signal}

Last, based on the mean values of parameters of five waves in Table I, a typical PTN-SEP signal and its five waves were synthesized, as illustrated in Fig. 7. We can see that the P37 and N45 components were very distinct in the synthetic PTN-SEP signal. Other small peaks and ridges in these synthetic waveforms were also labelled to facilitate further discussion on the associations between these waves and temporal components in the next section.

\section{DISCUSSION}

\section{A. Characteristics and Occurrences of SEP Waves}

In this paper, a mixture of TFA and clustering methods was employed to analyze the time-frequency features of SEP signals. A series of waves, which were in the form of Gabor functions and defined by five parameters, were identified in PTN-SEP signals of normal subjects. With the illustration of Fig. 7 and statistical data of Table I, we can describe a typical PTN-SEP response as follows. After a stimulus to the posterior tibial nerve, some short-latency responses (wave V) having largely different frequencies and amplitudes occur first ( $<30 \mathrm{~ms})$. At around $30-40 \mathrm{~ms}$ after the stimulus, some high-frequency $(80-120 \mathrm{~Hz})$ responses (wave II) are generated but their amplitudes are relatively small. Shortly after, the frequency of the SEP response decreases to about 40-60 $\mathrm{Hz}$ at around $40-50 \mathrm{~ms}$ but the fluctuation of magnitudes is much larger (wave I), resulting in two significant temporal components P37 and N45. After roughly $50 \mathrm{~ms}$, the SEP is mainly some low-frequency $(20-50 \mathrm{~Hz})$ middle-latency responses (waves III and IV), whose magnitudes are relatively large. Overall, a SEP signal is a non-stationary signal with time-variant frequency contents.

However, not all five waves could be identified in one individual SEP signal and the occurrences of waves II-V were respectively $34,32,18$, and 18 in 50 subjects. The low occurrence rates were due to several reasons. Firstly, we only considered the clustering of TFCs with relative energy larger than $1 \%$ and those TFCs with small energies were left out. Secondly, slight shifts for conducting potentials are very common between different trials of a SEP signal, so the signals under study, which were ensemble average of 100 trials, may lose some useful information. Thirdly, the heavy background noise influenced the SEP recordings and decomposition results. Of course, we cannot rule out the possibility that some waves are spurious and unstable. Among the five waves, the occurrence rates of waves IV and V 
Wave I
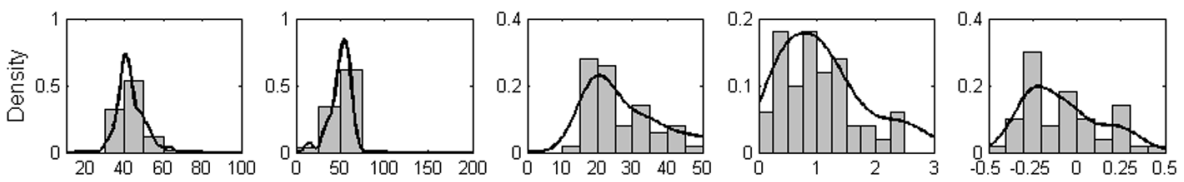

Wave II
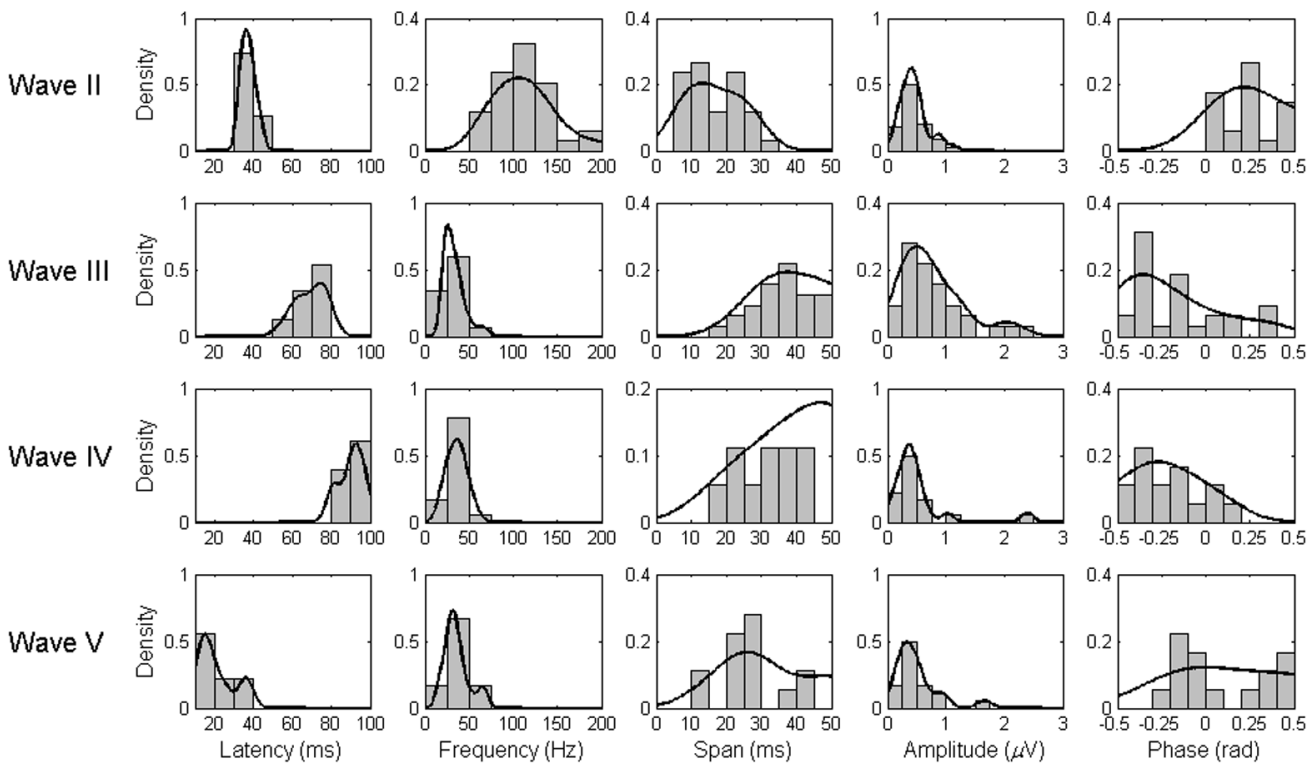

Fig. 6. Histograms and 1-D PDFs of MP parameters of five SEP waves. The histograms are normalized by the number of each cluster of TFCs.

were small and their average $\mathrm{CV}$ s were large, so the possibilities that they were spurious waves are relatively large.

\section{B. Associations Between SEP Waves and Conventional Features}

Next, we discuss the associations between the five waves and the temporal components of SEP. The SEP waveform components could consist of responses from nervous system and their volume conduction. When the electric stimulation was applied on posterior tibial nerve, several SEP responses could be recorded along the somatosensory pathway [28]. Typical peaks of those SEP waveforms include N8 for the popliteal fossa (PF) response, N22-N24 for spinal responses from L4/5 to C5 [29], P31 of sub-cortical response from $\mathrm{C} 5-\mathrm{Fz}$, and P37-N45 from cortical response $\mathrm{Cz}-\mathrm{Fz}$. The latency in different wave forms may help to indicate the origins of the components decomposed in this study. The temporal components P37 and N45 are two most important temporal components in PTN-SEP. As shown in Fig. 7, the components $I_{b}$ and $I_{c}$ of wave I were analogous to P37 and N45, respectively, although the rise between them was not as sharp as the rise from P37 to N45. The components $\mathrm{II}_{\mathrm{a}}$ and $\mathrm{II}_{\mathrm{b}}$ in wave $\mathrm{II}$, whose latencies were very close to the latencies of P37 and N45, also contributed a lot to the formation of P37 and N45. In addition, several peaks of waves I and II, including $\mathrm{I}_{\mathrm{a}}, \mathrm{II}_{\mathrm{a}}, \mathrm{II}_{\mathrm{b}}$, and $\mathrm{II}_{\mathrm{c}}$, were associated with some short-latency temporal components, such as $\mathrm{P} 30$ and N33 reported in [4]. On the other hand, waves III and IV (mainly the former) constituted the $\mathrm{W}$-shaped middle-latency response in a PTN-SEP signal [4]. It was difficult to find the counterparts of wave $\mathrm{V}$ in time domain. Although some peripheral components, such as $\mathrm{N} 8$ to N22 of PF and spinal responses originated from periphery nerve responses, had similar latency to the wave $\mathrm{V}$, they can hardly contribute to SEP signals recorded on the scalp. In addition, as mentioned before, the wave $\mathrm{V}$ had a low occurrence rate and its parameters exhibited a large variability. Considering all these factors, it is of great possibility that the wave $\mathrm{V}$ is a spurious component. The origin of wave $\mathrm{V}$ is not clear but its short latency suggests that it may be the stimulus artifact.

In summary, the relationship between waves I-V and temporal components of SEP can be concluded as 1) waves I and II formed the P37 and N45 components; 2) waves I and II contributed to several short-latency SEP components as well; 3) waves III and IV shaped the middle-latency W-shaped components.

The relationship and comparison between waves I-V and the peak identified in conventional TFA of SEP were also investigated. Wave I was the main TFC of a SEP signal and explained a large proportion of signal energy, which was similar to the main peak identified in TFD obtained by other TFA methods [9]-[12]. Table I showed that the latencies and frequencies of wave I were, respectively, $43.19 \pm 6.51 \mathrm{~ms}$ and $50.14 \pm 11.50 \mathrm{~Hz}$, while the peak times and peak frequencies of TFDs obtained by STFT with a 40-ms Hanning window were respectively 45.29 $\pm 6.98 \mathrm{~ms}$ and $40.77 \pm 4.16 \mathrm{~Hz}$. We can see that the wave I occurred a little earlier than the peak of STFT, and the frequency of wave I was higher than the peak frequency of STFT. The differences should be due to that, the peak in STFT was not only determined by wave I, but also influenced by other waves. In the synthetic SEP signal of Fig. 7, the relative energies of waves I-V were, respectively, $43.91 \%, 4.39 \%, 30.36 \%, 11.54 \%$, and $9.80 \%$. Two low-frequency waves (III and IV) had relatively larger energies and they lowered the frequency of the peak in 
TABLE I

Statistical Values of Parameters of Five SEP WaVes: The Values of Parameters are GiVen as Mean \pm SD (CV)

\begin{tabular}{|c|c|c|c|c|c|c|}
\hline Wave & I & II & III & IV & V & $\begin{array}{l}\text { Average CV of waves } \\
\text { for each parameter }\end{array}$ \\
\hline Latency (ms) & $\begin{array}{c}43.19 \pm 6.51 \\
(15.08)\end{array}$ & $\begin{array}{c}37.24 \pm 3.35 \\
(9.01)\end{array}$ & $\begin{array}{c}69.31 \pm 7.44 \\
(10.73)\end{array}$ & $\begin{array}{c}90.00 \pm 6.06 \\
(6.73)\end{array}$ & $\begin{array}{c}21.35 \pm 9.08 \\
(42.54)\end{array}$ & 16.82 \\
\hline Frequency $(\mathrm{Hz})$ & $\begin{array}{l}50.14 \pm 11.50 \\
(22.93)\end{array}$ & $\begin{array}{c}113.11 \pm 34.69 \\
(30.67)\end{array}$ & $\begin{array}{c}32.94 \pm 11.46 \\
(34.77)\end{array}$ & $\begin{array}{l}35.70 \pm 10.97 \\
(30.74)\end{array}$ & $\begin{array}{c}35.81 \pm 13.82 \\
(38.61)\end{array}$ & 31.54 \\
\hline Span (ms) & $\begin{array}{l}27.66 \pm 10.85 \\
(39.25)\end{array}$ & $\begin{array}{c}16.60 \pm 7.51 \\
(45.26)\end{array}$ & $\begin{array}{c}39.02 \pm 9.90 \\
(25.36)\end{array}$ & $\begin{array}{c}40.81 \pm 12.40 \\
(30.39)\end{array}$ & $\begin{array}{c}33.10 \pm 13.47 \\
(40.70)\end{array}$ & 36.19 \\
\hline Amplitude $(\mu \mathrm{V})$ & $\begin{array}{c}1.10 \pm 0.73 \\
(66.71)\end{array}$ & $\begin{array}{l}0.45 \pm 0.23 \\
(51.11)\end{array}$ & $\begin{array}{c}0.77 \pm 0.54 \\
(70.49)\end{array}$ & $\begin{array}{c}0.51 \pm 0.52 \\
(102.90)\end{array}$ & $\begin{array}{c}0.49 \pm 0.37 \\
(75.49)\end{array}$ & 73.34 \\
\hline Phase (rad) & $\begin{array}{c}-0.08 \pm 0.21 \\
(--)\end{array}$ & $\begin{array}{c}0.41 \pm 0.31 \\
(--)\end{array}$ & $\begin{array}{c}-0.21 \pm 0.27 \\
(--)\end{array}$ & $\begin{array}{c}-0.30 \pm 0.27 \\
(--) \\
\end{array}$ & $\begin{array}{c}0.20 \pm 0.33 \\
(--)\end{array}$ & -- \\
\hline $\begin{array}{c}\text { Average } \mathrm{CV} \text { of } \\
\text { parameters for each wave }\end{array}$ & 35.99 & 34.01 & 35.34 & 42.69 & 49.33 & \\
\hline
\end{tabular}

TABLE II

CORRELATION COEFFICIENTS BETWEEN PARAMETERS IN EACH SEP WAVE

\begin{tabular}{|c|c|c|c|c|c|c|c|c|c|}
\hline \multicolumn{5}{|c|}{ Wave I } & \multicolumn{5}{|c|}{ Wave II } \\
\hline \multirow[t]{5}{*}{$t$} & -0.15 & 0.16 & -0.01 & 0.43 & $t$ & -0.29 & 0.28 & 0.03 & -0.14 \\
\hline & $f$ & -0.1 & -0.59 & 0.03 & & $f$ & -0.20 & -0.51 & 0.00 \\
\hline & & $s$ & -0.24 & 0.27 & & & $s$ & -0.20 & -0.44 \\
\hline & & & $a$ & -0.05 & & & & $a$ & 0.22 \\
\hline & & & & $\phi$ & & & & & $\phi$ \\
\hline \multicolumn{5}{|c|}{ Wave III } & \multicolumn{5}{|c|}{ Wave IV } \\
\hline \multirow[t]{5}{*}{$t$} & -0.42 & -0.07 & $\begin{array}{l}-0.09 \\
\end{array}$ & -0.08 & $t$ & -0.38 & 0.50 & -0.37 & 0.32 \\
\hline & $f$ & 0.16 & -0.41 & 0.49 & & $f$ & 0.12 & -0.03 & 0.13 \\
\hline & & $s$ & -0.14 & 0.08 & & & $s$ & -0.12 & 0.21 \\
\hline & & & $a$ & -0.35 & & & & $a$ & 0.10 \\
\hline & & & & $\phi$ & & & & & $\phi$ \\
\hline \multicolumn{5}{|c|}{ Wave V } & & & & & \\
\hline \multirow[t]{5}{*}{$t$} & -0.29 & -0.17 & 0.02 & -0.19 & & & & & \\
\hline & $f$ & 0.19 & -0.34 & -0.2 & & & & & \\
\hline & & $s$ & -0.09 & -0.45 & & & & & \\
\hline & & & $a$ & 0.11 & & & & & \\
\hline & & & & $\phi$ & & & & & \\
\hline
\end{tabular}

STFT, while waves II and V were relatively smaller and contributed less to the peak. Overall, compared with the STFT, the proposed MP method can reveal more and finer time-frequency features of SEP.

As to the five parameters of the Gabor-type waves, we can see from Table I that the latency, which had a small variation $(\mathrm{CV}$ value), was the most stable and reliable parameter. The measurements of frequency and span were also suggestive and valuable, but the amplitude with a large variability was not very suitable as an indicator. The large variability of amplitude may stem from the considerable variability of scalp-electrode impedance and the inter-subject variability. Our observations on latency and amplitude of SEP waves were in good accordance with the conventional argument that the amplitudes of temporal components of SEP generally show larger variability than latencies [1].

\section{Limitations and Future Work}

This work proposed a new scheme consisting of high-resolution TFA method and pattern recognition method to discover minute time-frequency features of SEPs, and a more detailed description of time-frequency patterns of normal PTN-SEPs was presented based on the proposed scheme. The time and frequency locations and other parameters of the identified time-frequency components showed small intrasubjective variability, and thus these intrinsic and definite time-frequency
TABLE III

CORRELATION COEFFicientS BETWEEN WAVES FOR EACH PARAMETER

\begin{tabular}{|c|c|c|c|c|c|c|c|c|c|}
\hline \multicolumn{5}{|c|}{ Latency $t$} & \multicolumn{5}{|c|}{ Frequency $f$} \\
\hline I & 0.39 & -0.09 & -0.30 & 0.38 & I & 0.17 & 0.00 & -0.02 & -0.21 \\
\hline & II & -0.32 & 0.09 & 0.31 & & II & -0.15 & 0.01 & -0.30 \\
\hline & & III & 0.52 & 0.54 & & & III & -0.80 & -0.24 \\
\hline & & & IV & 0.21 & & & & IV & 0.73 \\
\hline & & & & $\mathrm{V}$ & & & & & $\mathrm{V}$ \\
\hline \multicolumn{5}{|c|}{ Span $s$} & \multicolumn{5}{|c|}{ Amplitude $a$} \\
\hline \multirow[t]{5}{*}{ I } & 0.21 & 0.19 & 0.00 & -0.06 & $\mathrm{I}$ & -0.09 & 0.11 & 0.37 & -0.31 \\
\hline & II & -0.14 & -0.32 & 0.87 & & II & -0.06 & 0.13 & 0.36 \\
\hline & & III & 0.69 & -0.21 & & & III & 0.47 & 0.17 \\
\hline & & & IV & 0.75 & & & & IV & -0.95 \\
\hline & & & & $\mathrm{V}$ & & & & & $\mathrm{V}$ \\
\hline \multicolumn{5}{|c|}{ Phase $\phi$} & & & & & \\
\hline I & -0.23 & 0.15 & 0.04 & -0.01 & & & & & \\
\hline & II & 0.04 & -0.59 & 0.42 & & & & & \\
\hline & & III & 0.06 & 0.49 & & & & & \\
\hline & & & IV & -0.33 & & & & & \\
\hline & & & & $\mathrm{V}$ & & & & & \\
\hline
\end{tabular}

features may provide important information for evaluation of somatosensory conduction and understanding the physiological mechanisms of SEP, which paves the way for future clinical and scientific research. It must be emphasised that the identified time-frequency components are used to represent the distribution of SEP power in the time-frequency domain. Thus, these time-frequency components are associated with typical power peaks of normal SEPs in the time-frequency domain, but they may not be strictly and individually associated with independent SEP components or waveforms having clear physiological and anatomical meaning (i.e., showing distinct spinal cord function or arising from specific sites in the nerve pathway). The study on the dependency between the five waves, as shown in Table III, suggested that some time-frequency components may have dependencies, such as waves III and IV. That is to say, an independent SEP component may be comprised of several Gabor-type time-frequency components. More precisely, all these identified time-frequency components provide an integrated description of normal PTN-SEPs in the time-frequency domain, but their individual physiological and anatomical meaning is still unknown or may be absent, which is the major limitation of this study.

The physiological meaning of the time-frequency features is expected to be demonstrated by investigating the relation between the time-frequency features and localised spinal cord 


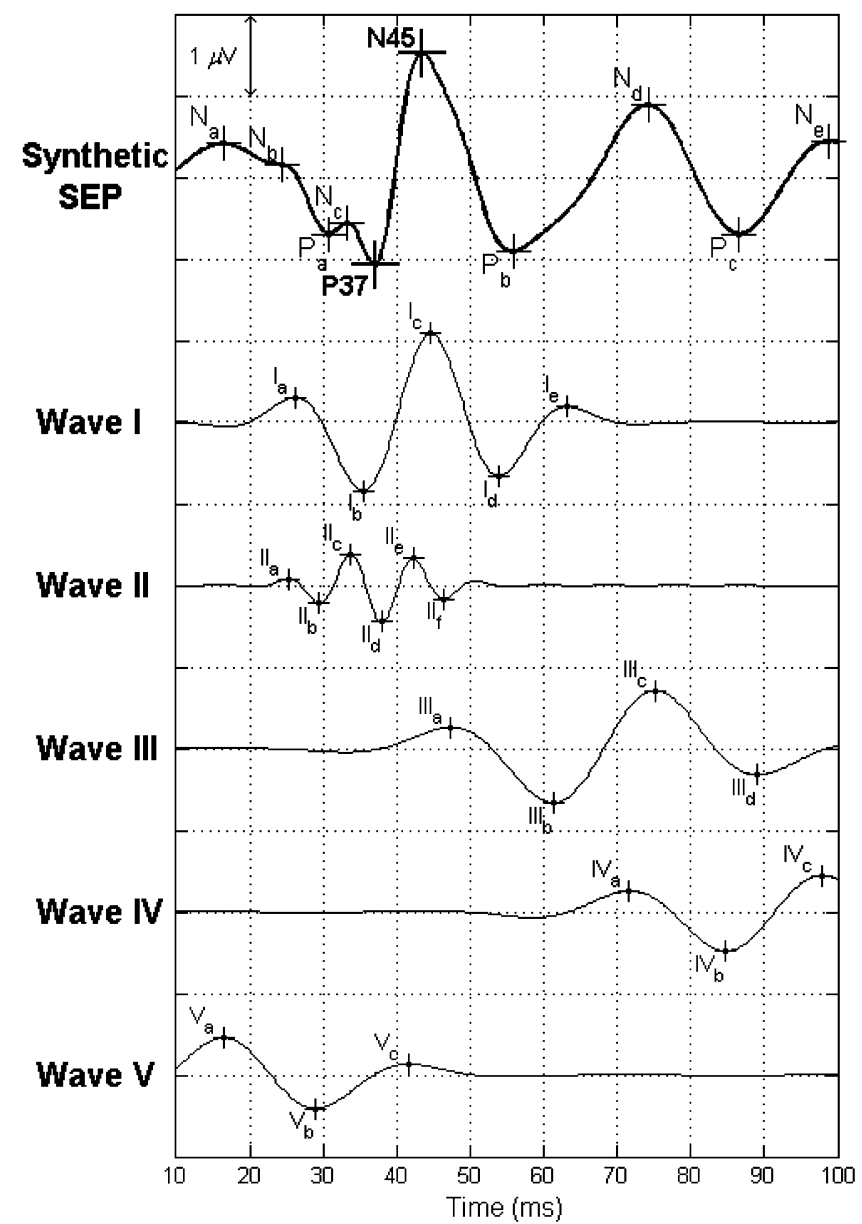

Fig. 7. A synthetic PTN-SEP signal of normal subjects and its five typical waves. Five synthetic waves were obtained using the mean values of parameters in Table I. The synthetic SEP waveform was obtained as the sum of waves I-V.

injury. Previous studies have shown that different SEP temporal components reflect different origins of spinal cord tracts [30]-[34]. Therefore, we hypothesize that these time-frequency features are jointly or individually associated with the localisation and pathological information of spinal cord function. In the study, the five typical SEP waves and the reference values for their parameters were obtained based on normal subjects without any spinal cord injury. When a specific injury occurs, these time-frequency features may exhibit certain change patterns. The occurrence and absence of SEP waves and the changes of their parameters can be used to indicate the changes of spinal cord functions. To evaluate this hypothesis, a new experimental study is being designed, which will incorporate well-defined focal spinal cord lesions and new animal models.

For clinical applications, this study provides a normal baseline of time-frequency features for assessment of the status of spinal cord during operation. However, the MP method has a very heavy computational complexity, which hinders its application to extract the time-frequency features of SEP during operations. Recently, several fast MP methods have been developed and some have been successfully applied in real-time video coding and audio analysis [35]-[37]. In future study, fast algorithms for the MP methods should be developed or applied for the purpose of spinal cord monitoring.
Lastly, the new time-frequency analysis scheme for SEPs provided in this paper can be further applied to analyze SEPs recorded over other locations and on a single-trial basis. Since current study only focuses on SEP recorded over scalp, it can not completely indicate the spinal cord function. Later, SEPs recorded over the spine and other locations should be analyzed to provide a more complete indicator of spinal cord function. In addition, a single-trial SEP recording contains richer information about complex brain dynamics than averaged SEP recording. Analyzing SEP recording on a single-trial basis with the proposed time-frequency analysis method may lead to more meaningful and interesting findings.

\section{CONCLUSION}

In this paper, the time-frequency features of SEP were studied by high-resolution time-frequency analysis and clustering methods. The MP algorithm first decomposed SEP signals into a set of TFCs, and a clustering was followed to group these TFCs into several clusters of waves. Five SEP waves were identified in PTN-SEP signals from 50 normal adults and they gave an integrated and detailed description of time-frequency features of SEP. The newly-identified time-frequency features of SEP may contribute to understanding complex response patterns and physiological origins of SEP. Further study on the correlation between these instinct components with the localisation and pathological information of spinal cord function may provide a new diagnostic tool for precision detection of spinal cord function deficits in clinical application.

\section{REFERENCES}

[1] K. H. Chiappa, Evoked Potentials in Clinical Medicine, 3rd ed. Philadelphia, PA: Lippincott-Raven, 1997.

[2] M. R. Nuwer, "Spinal cord monitoring with somatosensory techniques," J. Clin. Neurophysiol., vol. 15, no. 5, pp. 183-193, May 1998.

[3] J. S. Ebersole and T. A. Pedley, Current Practice of Clinical Electroencephalography, 3rd ed. Philadelphia, PA: Lippincott Williams Wilkins, 2003.

[4] E. Niedermeyer and F. H. Lopes da Silva, Electroencephalography: Basic principles, Clinical Applications, and Related Fields, 5th ed. Philadelphia, PA: Lippincott Williams Wilkins, 2005.

[5] T. P. Ryan and R. H. Britt, "Spinal and cortical somatosensory evoked potential monitoring during corrective spinal surgery with 108 patients," Spine, vol. 11, no. 4, pp. 352-361, May 1986.

[6] M. Sonoo, M. Kobayashi, K. Genba-Shimizu, T. Mannen, and T. Shimizu, "Detailed analysis of the latencies of median nerve somatosensory evoked potential components, 1: Selection of the best standard parameters and the establishment of normal values," Electroencephalogr. Clin. Neurophysiol., vol. 100, no. 4, pp. 319-331, July 1996.

[7] M. Sonoo, M. Kobayashi, K. Genba-Shimizu, T. Mannen, and T. Shimizu, "Detailed analysis of the latencies of median nerve somatosensory evoked potential components, 2: Analysis of subcomponents of the p13/14 and n20 potentials," Electroencephalogr. Clin. Neurophysiol., vol. 104, no. 4, pp. 296-311, Jul. 1997.

[8] J. C. Brauna, D. F. Hanley, and N. V. Thakor, "Detection of neurological injury using time-frequency analysis of the somatosensory evoked potential," Electroencephalogr. Clin. Neurophysiol., vol. 100, no. 4, pp. 310-318, Jul. 1996.

[9] Y. Hu, K. D. K. Luk, W. W. Lu, A. Holmes, and J. C. Leong, "Prevention of spinal cord injury with time-frequency analysis of evoked potentials: An experimental study," J. Neurol. Neurosurg. Psychiatry, vol. 71, no. 6, pp. 732-740, Jun. 2001

[10] Y. Hu, K. D. K. Luk, W. W. Lu, A. Holmes, and J. C. Leong, "Comparison of time-frequency distribution techniques for analysis of spinal somatosensory evoked potential," Med. Biol. Eng. Comput., vol. 39, no. 3, pp. 375-380, May 2001. 
[11] Y. Hu, K. D. K. Luk, W. W. Lu, and J. C. Leong, "Comparison of timefrequency analysis techniques in intraoperative somatosensory evoked potential (SEP) monitoring," Comput. Biol. Med., vol. 32, no. 1, pp. 13-23, Jan. 2002.

[12] Y. Hu, K. D. K. Luk, W. W. Lu, and J. C. Leong, "Application of time-frequency analysis to somatosensory evoked potential for intraoperative spinal cord monitoring," J. Neurol. Neurosurg. Psychiatry, vol. 74, no. 1, pp. 82-87, Jan. 2003.

[13] N. V. Thakor, X. R. Guo, Y. C. Sun, and D. F. Hanley, "Multiresolution wavelet analysis of evoked potentials," IEEE Trans. Biomed. Eng., vol. 40, no. 11, pp. 1085-1094, Nov. 1993.

[14] A. B. Geva, H. Pratt, and Y. Y. Zeevi, "Multichannel wavelet-type decomposition of evoked potentials: Model-based recognition of generator activity," Med. Biol. Eng. Comput., vol. 35, no. 1, pp. 40-46, Jan. 1997.

[15] U. Hoppe, K. Schnabel, S. Weiss, and I. Rundshagen, "Representation of somatosensory evoked potentials using discrete wavelet transform," J. Clin. Monit. Comput., vol. 17, no. 3-4, pp. 227-233, Apr.-May 2002.

[16] S. G. Mallat and Z. Zhang, "Matching pursuits with time-frequency dictionaries," IEEE Trans. Signal. Process., vol. 41, no. 12, pp. 3397-3415, Dec. 1993.

[17] P. J. Durka, Matching Pursuit and Unification in EEG Analysis. Boston, MA: Artech House, 2007.

[18] W. W. Jedrzejczak, K. J. Blinowska, and W. Konopka, "Time-frequency analysis of transiently evoked otoacoustic emissions of subjects exposed to noise," Hear. Res., vol. 205, no. 1-2, pp. 249-255, Jul. 2005.

[19] J. Cui and W. Wong, "Investigation of short-term changes in visual evoked potentials with windowed adaptive chirplet transform," IEEE Trans. Biomed. Eng., vol. 55, no. 3, pp. 1449-1454, Apr. 2008.

[20] R. O. Duda, P. E. Hart, and D. G. Stork, Pattern Classification, 2nd ed. New York: Wiley, 2001.

[21] K. D. K. Luk, Y. Hu, W. W. Lu, and Y. W. Wong, "Effect of stimulus pulse duration on intraoperative somatosensory evoked potential (SEP) monitoring," J. Spinal Disord., vol. 14, no. 2, pp. 247-251, Jun. 2001.

[22] M. A. Carreira-Perpinan, A review of dimension reduction techniques Univ. Sheffield, Sheffield, U.K., Tech. Rep. CS-96-09, 1997.

[23] A. K. Jain, M. N. Murty, and P. J. Flynn, "Data clustering: A review," ACM Comp. Surv., vol. 31, no. 3, pp. 264-323, 1999.

[24] P. S. Bradley and U. M. Fayyad, "Refining initial points for K-means clustering," in Proc. 15th Int. Conf. Mach. Learn., 1998, pp. 91-99.

[25] J. M. Peña, J. A. Lozano, and P. Larrañaga, "An empirical comparison of four initialization methods for the K-means algorithm," Pattern Recognit. Lett., vol. 20, no. 10, pp. 1027-1040, Oct. 1999.

[26] Q. T. Zeng, J. P. Pratt, J. Pak, D. Ravnic, H. Huss, and S. J. Mentzer, "Feature-guided clustering of multi-dimensional flow cytometry datasets," J. Biomed. Inform., vol. 40, no. 3, pp. 325-331, Jun. 2007.

[27] A. W. Bowman and A. Azzalini, Applied Smoothing Techniques for Data Analysis: The Kernel Approach With S-Plus Illustrations. New York: Oxford Univ. Press, 1997.

[28] M. R. Nuwer, M. Aminoff, J. Desmedt, A. A. Eisen, D. Goodin, S. Matsuoka, F. Mauguière, H. Shibasaki, W. Sutherling, and J. F. Vibert, "IFCN recommended standards for short latency somatosensory evoked potentials. Report of an IFCN committee. International Federation of Clinical Neurophysiology," Electroencephalogr. Clin. Neurophysiol., vol. 91, no. 1, pp. 6-11, Jul. 1994.

[29] R. M. Nuwer, Evoked Potential Monitoring in the Operating Room. New York: Raven, 1986, p. 50.

[30] H. Lueders, D. S. Dinner, R. P. Lesser, and G. Klem, "Origin of farfield subcortical evoked potentials to posterior tibial and median nerve stimulation. A comparative study," Arch. Neurol., vol. 40, no. 2, pp. 93-97, Feb. 1983.
[31] E. Urasaki, S. Wada, A. Yokota, T. Tokimura, and H. Yasukouchi, "Origin of subcortical somatosensory evoked potentials in response to posterior tibial nerve stimulation in humans," J. UOEH, vol. 15, no. 2, pp. 113-135, Jun. 1993.

[32] G. Zanette, M. Tinazzi, A. Polo, and N. Rizzuto, "Motor neuron disease with pyramidal tract dysfunction involves the cortical generators of the early somatosensory evoked potential to tibial nerve stimulation," $\mathrm{Neu}$ rology, vol. 47, no. 4, pp. 932-938, Oct. 1996.

[33] E. K. Lee and M. Seyal, "Generators of short latency human somatosensory-evoked potentials recorded over the spine and scalp," $J$. Clin. Neurophysiol., vol. 15, no. 3, pp. 227-234, May 1998.

[34] V. Deletis and F. Sala, "Intraoperative neurophysiological monitoring of the spinal cord during spinal cord and spine surgery: A review focused on the corticospinal tracts," Clin. Neurophysiol., vol. 119, pp. 248-264, 2008.

[35] R. Gribonval, "Fast matching pursuit with a multiscale dictionary of Gaussian chirps," IEEE Trans. Signal Process., vol. 49, no. 5, pp. 994-1001, May 2001.

[36] J.-L. Lin, W.-L. Hwang, and S.-C. Pei, "Multiple blocks matching pursuit update algorithm for low bit rate video coding," IEEE Trans. Circuits Syst. Video Technol., vol. 16, no. 3, pp. 331-337, Mar. 2006.

[37] S. Strahla and A. Mertins, "Sparse gammatone signal model optimized for English speech does not match the human auditory filters," Brain Res., vol. 1220, pp. 224-233, July 2008.

Zhiguo Zhang (M'07) received the B.Sc. degree in electronic and information engineering from Tianjin University, China, in 2000, and the M.Eng. degree in electronic engineering and information science from the University of Science and Technology of China, Hefei, China, in 2003. He received the Ph.D. degree in 2008 from the Department of Electrical and Electronic Engineering at the University of Hong Kong, where he is currently a Postdoctoral Fellow.

He worked as a Research Assistant in the Department of Orthopaedics and Traumatology, The University of Hong Kong from 2007 to 2008. His research interests are in general area of statistical signal processing and digital signal processing, and biomedical signal processing.

Keith D. K. Luk graduated from the University of Hong Kong, in 1977. He received the FRCS, in 1981, MCh Orth from the University of Liverpool, in 1984, and the FRACS, in 1985.

He is Chair Professor and Head of the Department of Orthopaedics and Traumatology, The University of Hong Kong. His main clinical and research interest is in spinal surgery.

Yong Hu (M'07) received the B.Sc. and M.Sc degrees in biomedical engineering from Tianjin University, Tianjin, China, in 1985 and 1988, respectively, the Ph.D. degree from The University of Hong Kong, in 1999.

He is currently an Assistant Professor in the Department of Orthopaedics and Traumatology, The University of Hong Kong. He is a distinguished adjunct professor in Institute of Biomedical Engineering, Chinese Academy of Medical Sciences and Peking Union Medical College. His research interests include neural engineering, clinical electrophysiology, biomedical signal measurement and processing. 